\title{
Operational algorithm for ice-water classification on dual-polarized RADARSAT-2 images
}

\author{
Natalia Zakhvatkina ${ }^{1,2}$, Anton Korosov ${ }^{3}$, Stefan Muckenhuber ${ }^{3}$, Stein Sandven $^{3}$, and Mohamed Babiker ${ }^{3}$ \\ ${ }^{1}$ Nansen International Environmental and Remote Sensing Centre (Nansen Centre, NIERSC), 14th Line 7, \\ Office 49, Vasilievsky Island, St. Petersburg, 199034, Russian Federation \\ ${ }^{2}$ Arctic and Antarctic Research Institute (AARI), Bering Str. 38, St. Petersburg, 199397, Russian Federation \\ ${ }^{3}$ Nansen Environmental and Remote Sensing Center (NERSC), Thormøhlensgate 47, 5006 Bergen, Norway \\ Correspondence to: Natalia Zakhvatkina (natalia.piotrovskaya@niersc.spb.ru)
}

Received: 30 May 2016 - Published in The Cryosphere Discuss.: 21 June 2016

Revised: 23 October 2016 - Accepted: 6 December 2016 - Published: 11 January 2017

\begin{abstract}
Synthetic Aperture Radar (SAR) data from RADARSAT-2 (RS2) in dual-polarization mode provide additional information for discriminating sea ice and open water compared to single-polarization data. We have developed an automatic algorithm based on dual-polarized RS2 SAR images to distinguish open water (rough and calm) and sea ice. Several technical issues inherent in RS2 data were solved in the pre-processing stage, including thermal noise reduction in HV polarization and correction of angular backscatter dependency in $\mathrm{HH}$ polarization. Texture features were explored and used in addition to supervised image classification based on the support vector machines (SVM) approach. The study was conducted in the ice-covered area between Greenland and Franz Josef Land. The algorithm has been trained using 24 RS2 scenes acquired in winter months in 2011 and 2012, and the results were validated against manually derived ice charts of the Norwegian Meteorological Institute. The algorithm was applied on a total of 2705 RS2 scenes obtained from 2013 to 2015 , and the validation results showed that the average classification accuracy was $91 \pm 4 \%$.
\end{abstract}

\section{Introduction}

Synthetic Aperture Radar (SAR) is an active microwave sensor providing high-resolution images over large areas independent of clouds and daylight. This is especially useful for observing the polar regions, where SAR data are widely used for exploring sea ice concentration, extent, detection of leads, polynyas, ice floes and ice edge, and ice type identification and classification (Johannessen et al., 2007; Dierking, 2013). Monitoring of sea ice processes, i.e., ice edge variations and motion, is important for practical tasks such as ice navigation and for scientific studies. High-resolution data from C-band SAR such as ERS-1/2 (European Remote Sensing satellites, European Space Agency, ESA), RADARSAT-1 (Earth observation satellite, Canadian Space Agency), and ENVISAT (Environmental Satellite, ESA) have been used as the main data source for sea ice monitoring in the last 2 decades (e.g., Johannessen et al., 2007). The advanced capabilities of SAR on board of RADARSAT-2 (RS2) and Sentinel-1 (European Commission and ESA) with multi-polarization data can improve sea ice observations such as ice edge detection and ice type classification.

SAR images can be used to identify different sea ice types and open water (OW) areas based on variations of the backscattered radar intensity caused by surface roughness and other sea ice properties. Classification methods based only on the backscattering coefficients $\left(\sigma^{\circ}\right)$ are hampered by ambiguities in the relation between ice types and $\sigma^{\circ}$, since various ice types (multiyear, first-year, and some young and new ice) and open water depending on wind speed and direction can have similar $\sigma^{\circ}$ (Dierking, 2010; Johannessen et al., 2007). In particular, discrimination between calm open water and smooth first-year ice, as well as between windy open water and young ice with frost flowers or multiyear ice, can be problematic. Including additional image characteristics like image texture, tone, and spatial structures can improve the classification results significantly (Shokr, 1991; Soh and 
Tsatsoulis, 1999; Clausi, 2002; Bogdanov et al., 2005; Maillard et al., 2005; Yu et al., 2012).

Numerous efforts have been made to develop algorithms to retrieve sea ice variables from SAR data. The SAR polynya detection algorithm proposed by Dokken et al. (2002) is based on wavelet transforms for edge detection and standard texture analysis. A threshold function using texture information is used to classify sea ice and water for polynya detection. A semi-automated sea ice classification method based on fuzzy rules was reported by Gill (2003) for classification of RADARSAT-1 data over the Arctic into calm water, windroughened water, and sea ice in low and high concentrations. Advanced Reasoning using Knowledge for Typing of Sea Ice (ARKTOS) (Soh et al., 2004) has been established to support scientific research and operational applications in the field of sea ice segmentation and classification. Haarpainter and Solbø (2007) developed an automatic algorithm for iceocean discrimination in RADARSAT- 1 and ENVISAT SAR imagery. The texture-based algorithm consists of an automatically trained maximum likelihood classifier and divides the SAR images into slices of small incidence angle ranges. The results show that sea ice and water can be discriminated quite reliably. Some examples showed a tendency of the algorithm to a better performance at low incidence angles. Karvonen et al. (2005) distinguished the Baltic Sea ice from open water based on thresholding of segment-wise local autocorrelations in SAR images. The method provided $90 \%$ accuracy compared to digital ice charts for the Baltic Sea. This algorithm has been used by the Finnish Meteorological Institute (FMI). Tests with RADARSAT- 2 and ENVISAT SAR data show that over $89.4 \%$ of the test data fit the ice classification provided by the Finnish Ice Service for the Baltic Sea and Arctic Sea (Karvonen, 2010, 2012).

Dual polarization has several advantages for sea ice classification compared to single-polarization SAR data. Rough or frost-flower-covered young ice and multiyear ice, while very different in their thickness $(10-15 \mathrm{~cm}$ and more than $2.5 \mathrm{~m}$, respectively), show rather similar brightness in the HH channel whereas MYI is brighter than young ice in the HV channel. Smooth first-year level ice is darker in both $\mathrm{HH}$ and HV and can be easily distinguished from young ice and MYI. Wind-roughened open water is difficult to distinguish from sea ice in a single $\mathrm{HH}$ polarization. However, open water especially affected by wind is darker in HV that improves sea ice classification (Sandven et al., 2008). The dual-polarization ENVISAT SAR Alternative Polarization Mode data enabled discrimination of sea ice types and open water with a decision-tree classifier using estimated statistical thresholds for winter. Open water can be unambiguously discriminated from smooth FYI, rough FYI, and MYI with $>99 \%$ accuracy using a co-polarized ratio threshold (Geldsetzer and Yackel, 2009). The possibilities of supervised $k$-means and maximum likelihood classification of various SAR polarimetric data to three pre-identified sea ice types and wind-roughened open water was explored in Gill and Yackel (2012).

The MAp-Guided Sea Ice Classification System (MAGIC) for automated ice-water discrimination on dual-polarization images from RADARSAT-2 combines a "glocal" Iterative Region Growing using Semantics (IRGS) classification (Yu and Clausi, 2008) with a pixel-based support vector machine (SVM) approach. The "glocal" classification identifies homogeneous regions with arbitrary class labels. The ice-water map is created with the SVM classifier exploiting SAR texture and backscatter features. The MAGIC system has been applied on 20 RS2 scenes over the Beaufort Sea. The average classification accuracy with respect to manually drawn ice charts is $96.5 \%$ (Clausi et al., 2010; Ochilov and Clausi, 2012; Leigh et al., 2014).

A neural-network-based algorithm has been developed for ENVISAT SAR images for operational sea ice classification including validation (Zakhvatkina et al., 2013). The algorithm discriminated the level FYI, deformed FYI, MYI, and open water/nilas in the high Arctic in winter conditions and demonstrated good applicability in the central Arctic. Using the same approach an algorithm for mapping icewater utilizing ENVISAT ASAR WSM images was created for automated ice edge detection in Fram Strait. The icewater classes were estimated by a multi-layer perceptron neural network which uses SAR calculated texture features and concentration data from AMSR (Advanced Microwave Scanning Radiometer) and, later, SSM/I (Special Sensor Microwave/Imager) as inputs (Sandven et al., 2012). Daily icewater products were provided with a resolution of $525 \mathrm{~m}$ from winter 2011 until April 2012. The accuracy of this classification was about $97 \%$ compared to high-resolution sea ice concentration charts based on manual interpretation of satellite data provided by the Norwegian Meteorological Institute.

Our goal is to extend the method originally used for the single polarized ENVISAT SAR images (Sandven et al., 2012) by utilizing dual-polarization data from RS2 and to develop an algorithm for ice-water classification, which can be applied to RS2 data for the production of ice-water maps as part of marine services under the Copernicus programme. A special motivation for our work was not only development of an algorithm but also its extensively validation in various sea ice conditions and identification of the applicability conditions. We also aimed to develop the algorithm as an opensource software available for other scientists. Our algorithm is based on texture features and the SVM method using the advantages of dual-polarization RS2 SAR image data.

This paper describes the developed algorithm and discusses practical issues of its applicability. The steps and parameters for implementation of the algorithm are described, allowing users to test the algorithms themselves. The paper is organized as follows. Section 2 introduces the satellite images and geographical area used in the study. The algorithm including pre-processing and validation procedure is 
described in Sect. 3. Results of the pre-processing step, icewater classification, and comparison with manual ice charts are given in Sect. 4. Finally, a discussion of the results is presented in Sect. 5.

\section{Data}

The region of interest is the ice-covered sea between Greenland and Franz Josef Land, where detailed ice information from SAR data is important due to the highly variable sea ice conditions, in particular the export out of the Arctic through Fram Strait (Vinje and Finnekåsa, 1986). SAR is the most useful sensor to provide high-resolution year-round data for estimation of sea ice variables such as ice classification, ice edge variability, and ice drift.

This study is based on RS2 ScanSAR Wide (SCW) mode images with $500 \mathrm{~km}$ swath width, a pixel spacing of $50 \times 50 \mathrm{~m}$, and dual-polarization $(\mathrm{HH}+\mathrm{HV})$. This is the main mode used by RS2 for operational sea ice monitoring (RS2 Product Description, 2011). Twenty-four SCW scenes around Svalbard (Fig. 1) from 2011 and 2012 were utilized in the following analysis to train the algorithm. The wintermonth images were selected to cover various types of thin (e.g., new and young ice), first-year, and multiyear ice with different degrees of deformation, packed ice, broken ice, and open water under different wind speed conditions (rough, very rough, and calm water, also in leads). The radar images include the most typical samples since the radar intensity contrast between open water and ice varies greatly with ice conditions and wind speed or direction which significantly affect the radar brightness of open water. In summer the contrast between backscatter intensities of the melted different ice types observed on the SAR image is diminished since surfaces become smoother and are covered by meltwater. The intensities are reduced as well as the contrast between ice and OW.

The backscatter at $\mathrm{HH}$ generally decreases with increasing incidence angle (Fig. 2a), whereas the HV channel is less sensitive to the incidence angle. The HV channel includes disturbances in azimuth direction (visible as bright and dark stripes) along the burst boundaries in the ScanSAR Wide Beam SAR image (Fig. 2b). The expected noise level is a local mean noise power value that fluctuates across the image. The noise level is obtained from a model that accounts for the characteristics of the SAR sensor, the beam mode, the acquisition, and the ground processing (RS2 PUG) (Jefferies, 2012). The system noise level as a function of the incidence angle is documented in the XML file that comes with the RS2 image.

\section{Methodology}

\subsection{Incidence angle correction for $\mathrm{HH}$}

During the first step of our ice-water classification algorithm SAR data pre-processing is conducted, including incidence angular correction for $\mathrm{HH}$ and absolute calibration to obtain $\sigma^{\circ}$ values. The auxiliary XML files coming with the product, i.e., scaling look-up table (LUT), provide information for georeferencing and calibration. These LUTs allow converting the processed digital numbers of the output SAR image to calibrated values. An important goal of radiometric calibration is to provide the proper comparison between the scattering of image targets with different SAR sensors or from the same sensor with different operating conditions, so the backscatter values of targets can be compared to one another or a reference. Absolute radiation calibration is used to convert the digital numbers in the SAR image to $\sigma^{\circ}$, applying a constant offset and range dependent gains to the SAR image (RS2 Product Description, 2011). All images are corrected to a reference angle of $35^{\circ}$, which represents the center incidence angle and allows analysis of the SAR images without brightness amplification. Backscatter recalculation to $35^{\circ}$ incidence angle is carried out using a predefined calculated coefficient:

$$
\begin{aligned}
\sigma_{j}^{\circ} & =10 \cdot \log _{10}\left(\frac{\left(\text { digital number }_{j}^{2}\right)}{A_{j}} \cdot \sin \left(\theta_{j}\right)\right) \\
& -\left(\text { coefficient } \cdot\left(\theta_{j}-35\right)\right),
\end{aligned}
$$

where $\sigma^{\circ}$ is the backscatter values of pixels in $j$ th line (range direction), given in $\mathrm{dB}$; digital number is the pixel brightness (data consist of the SAR amplitude value Amp and intensity value $I=\mathrm{Amp}^{2}$; $A$ is the gain value (invariant in line) corresponding to the range sample $j$ (obtained by linear interpolation of the LUT supplied gain values); $\theta$ is the incidence angle for each jth pixel; and coefficient is the predefined calculated coefficient.

The coefficient was defined by calculating the linear trend of the observed backscatter signal on several HH-polarized RS2 SCW images of pack ice. The procedure is similar to the pre-processing of ENVISAT ASAR data in Zakhvatkina et al. (2013). The backscatter normalization to a pre-defined incidence angle provides homogenous image contrast across the swath over ice-covered areas. The details of the angular correction method are discussed in Sect. 5.1.

\subsection{Thermal noise correction for $\mathbf{H V}$}

SAR data pre-processing also includes reduction of thermal noise effect and absolute calibration for HV. The thermal noise reduction consists of three steps: (1) reading the noise values and corresponding incidence angles from the XML file, (2) interpolation of noise on a finer grid for each 


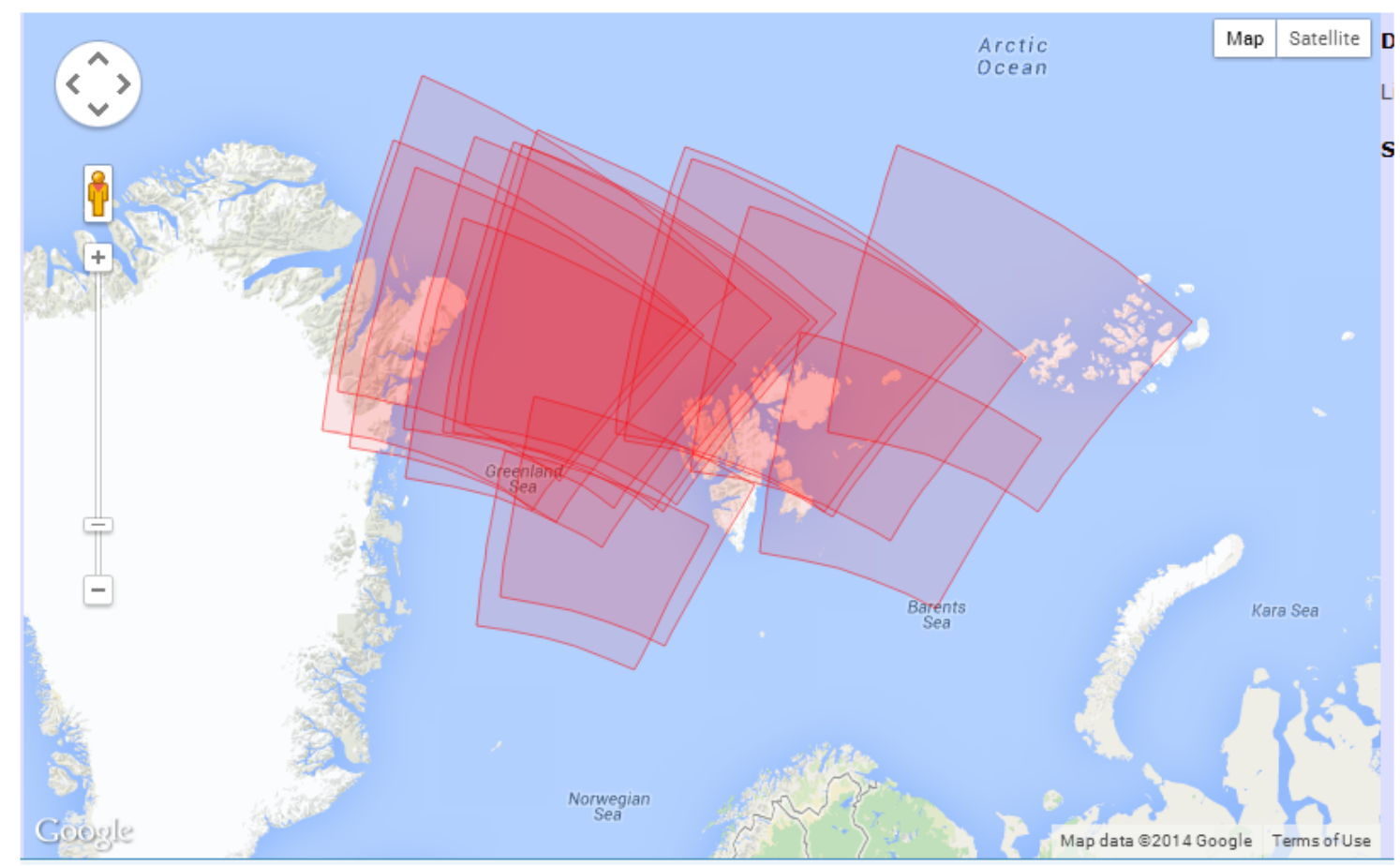

Figure 1. Location of RADARSAT-2 image used for training. All data are provided in GeoTIFF format with auxiliary XML files.

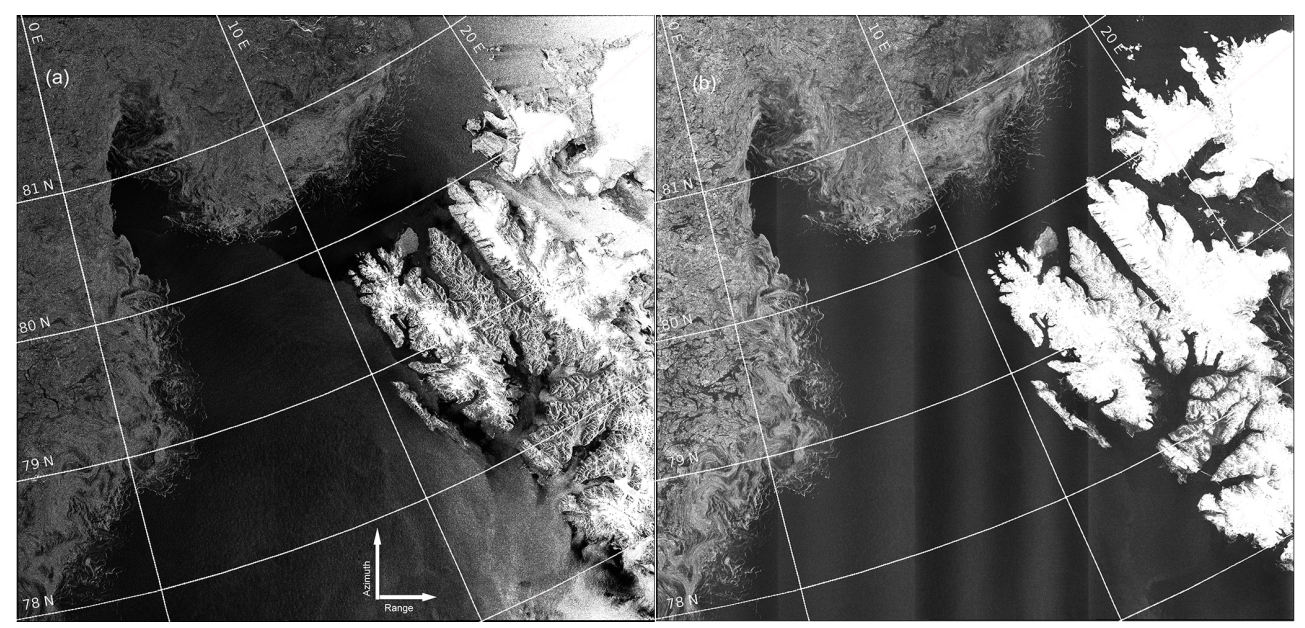

Figure 2. RS2 SCW dual-polarization image taken over Fram Strait on 28 November 2011 prior pre-processing. (a) HH channel with angular dependence; (b) HV channel with noise floor variations.

pixel, and (3) subtraction of interpolated noise values from the backscatter values of the entire image.

Due to the discontinuity of the noise floor at the boundaries of the individual SAR beams and the low resolution of the provided noise values in the XML file (only 100 points for $500 \mathrm{~km}$ swath width), the noise correction may result in an erroneous subtraction of a high noise floor from a low signal of the neighboring SAR beam and, hence, yield negative values for $\sigma^{\circ}$. To prevent such flaws, a 10 pixel wide stripe of data along the edge of the SAR beam is masked out and disabled for further analysis.

\subsection{Manual classification}

The second step includes manual classification of SAR images into predefined classes (e.g., open water and ice of various types depending on which classes are needed). The predefined classes take into account information from optical data, ice concentration from passive microwave, previous classification results, and historical data. 
Manual classification has been done for the training images containing several different sea ice types and ice-free areas with both rough and smooth open water. Predominant subclasses, which must be reliable and of high quality, were identified and chosen by sea ice experts through visual analysis of RS2 scenes based on their previous experience. The images selected for our algorithm training did not contain homogeneous ice cover because the mixing of different ice types with different degrees of deformation, cracks, ridges and leads usually occurs in ice-covered areas. The main class "sea ice" was chosen to include the following subclasses: (1) subclass including young ice, first-year, and multiyear ice; (2) fast ice; and (3) broken ice on the edge (border) mixed with ice-free areas (mostly found in the marginal ice zone). The class "open water" included the two subclasses open water with high and very high wind speed conditions and a third subclass that represented a mixture of calm open water, frazil ice, leads, and nilas. These manual classification results were collocated with texture feature images (description provided in Sects. 3.4 and 4.2) to get a number of training vectors. For the final product the subclasses were merged into the main classes "sea ice" and "open water" since the similarities between the subclasses are too high for a reliable discrimination without additional data.

\subsection{Calculation of texture features}

The third step is a calculation of texture features from $\mathrm{HH}$ and HV images. The calculation of texture features consists of the computation of the gray level co-occurrence matrix (GLCM) using Eq. (2) and the calculation of texture features based on the GLCM (Eqs. 3-10). Considering the full range of possible brightness levels (e.g., 0-255) and a small window size, most GLCM elements would be zero and that would have a negative effect on the classification result. Therefore we divide the full brightness range into few intervals (quantization levels $K$ ). The GLCM is created for each direction $\theta$, where each cell $(i, j)$ is a measure of the relative frequency of two pixels occurrence with brightness $i$ and $j$, respectively, separated by a co-occurrence distance $d$. One may also say that the matrix element $P_{d, \theta}(i, j)$ is a measure of the second-order statistical probability for changes between gray levels $i$ and $j$ at a particular displacement distance $d$ and at a particular angle (direction) $(\theta)$. The size of square GLCM is equal to number of quantized brightness levels $K$. The GLCM is averaged over four directions $\theta$ (0, $45,90,135^{\circ}$ ) to account for possible rotation of the ice floes (Clausi, 2002; Haralick et al., 1973).

$S_{d, \theta}(i, j)=\frac{P_{d, \theta}(i, j)}{\sum_{i=1}^{K} \sum_{j=1}^{K} P_{d, \theta}(i, j)}$,

where $S_{d, \theta}$ is the GLCM, $P_{d, \theta}$ is the number of neighbor pixel pairs, $\theta$ is the fixed vector directions $\left(0,45,90,135^{\circ}\right)$, $d$ is the co-occurrence distance, $K$ is the number of quantized gray levels, and $i, j$ are the gray levels (0-255).

$$
\begin{aligned}
& \text { Energy }=\sum_{i=1}^{K} \sum_{j=1}^{K}\left[S_{d, \theta}(i, j)\right]^{2} \\
& \text { Homogeneity }=\sum_{i=1}^{K} \sum_{j=1}^{K} \frac{S_{d, \theta}(i, j)}{1+(i-j)^{2}} \\
& \text { Contrast }=\sum_{i=1}^{K} \sum_{j=1}^{K}(i-j)^{2} S_{d, \theta}(i, j) \\
& \text { Correlation }=\frac{\sum_{i=1}^{K} \sum_{j=1}^{K}\left(i-\mu_{x}\right)\left(j-\mu_{y}\right) S_{d, \theta}(i, j)}{\sigma_{x} \sigma_{y}} \\
& \text { Entropy }=-\sum_{i=1}^{K} \sum_{j=1}^{K} S_{d, \theta}(i, j) \log _{10} S_{d, \theta}(i, j) \\
& \text { Kurtosis }=\sum_{i=1}^{K} \sum_{j=1}^{K} \frac{\left(S_{d, \theta}-\mu\right)^{4}}{\sigma^{4}} \\
& \text { Skewness }=\sum_{i=1}^{K} \sum_{j=1}^{K} \frac{\left(S_{d, \theta}-\mu\right)^{3}}{\sigma^{3}}
\end{aligned}
$$

Cluster prominence $=$

$\sum_{i=1}^{K} \sum_{j=1}^{K}\left(i+j-\mu_{x}-\mu_{y}\right)^{4} S_{d, \theta}(i, j)$

$\sigma_{x}^{2}=\sum_{i=1}^{K} \sum_{j=1}^{K}\left(j-\mu_{x}\right)^{2} S_{d, \theta}(i, j) \quad$ and $\quad \sigma_{y}^{2}=$ $\sum_{i=1}^{K} \sum_{j=1}^{K}\left(j-\mu_{y}\right)^{2} S_{d, \theta}(i, j) \quad$ are $\quad$ standard deviation of rows and columns, $\mu_{x}=\sum_{i=1}^{K} \sum_{j=1}^{K} i S_{d, \theta}$ and $\mu_{y}=\sum_{i=1}^{K} \sum_{j=1}^{K} j S_{d, \theta}$ are mean values of rows and columns, $\sigma^{2}=\sum_{i=1}^{K}(i-\mu)^{2} \sum_{j=1}^{K} S_{d, \theta}(i, j)$ is the standard deviation, and $\mu=\sum_{i=1}^{K} \sum_{j=1}^{K} i S_{d, \theta}(i, j)$ is the mean values of brightness.

The results of this procedure depend on several factors such as the size of the sliding window, the co-occurrence distance, and the quantization levels (Shokr, 1991; Soh and Tsatsoulis, 1999; Clausi, 2002). In order to test the effects of these parameters on the classification accuracy, texture features were calculated for the window sizes 16, 32, 64, and 128 pixels using different co-occurrence distances and varying the number of quantized gray levels (Table 1). The optimal values for the parameters of texture features calculation were selected analyzing variations in the texture parameters by visual inspection of the normalized mean values distribution of each texture feature for a defined class. The decision is made for the benefit of the cases when the separation of the normalized texture values for the classes increases in the majority of investigated texture feature figures. Defined parameters were applied for calculations of all set of texture features, and then the visual comparison showed the best discrimination between the ice-water classes for some texture features (details provided in Sect. 4.2). 
Table 1. Experiments of computation parameters. $W$ is the window size, $d$ is the co-occurrence distance, $K$ is the quantized gray level, and moving step is a step of sliding window moving.

\begin{tabular}{lrrr}
\hline$W$ & $d$ & Moving step & $K$ \\
\hline 32 & 4 & $8 / 16 / 32$ & $16 / 25 / 32$ \\
32 & 8 & $8 / 16 / 32$ & $16 / 25 / 32$ \\
32 & 16 & $8 / 16 / 32$ & $16 / 25 / 32$ \\
64 & 4 & $8 / 16 / 32 / 64$ & $16 / 25 / 32$ \\
64 & 8 & $8 / 16 / 32 / 64$ & $16 / 25 / 32$ \\
64 & 16 & $8 / 16 / 32 / 64$ & $16 / 25 / 32$ \\
64 & 32 & $8 / 16 / 32 / 64$ & $16 / 25 / 32$ \\
128 & 4 & $32 / 64 / 128$ & $16 / 25 / 32$ \\
128 & 8 & $32 / 64 / 128$ & $16 / 25 / 32$ \\
128 & 16 & $32 / 64 / 128$ & $16 / 25 / 32$ \\
128 & 32 & $32 / 64 / 128$ & $16 / 25 / 32$ \\
128 & 64 & $32 / 64 / 128$ & $16 / 25 / 32$ \\
\hline
\end{tabular}

A selection procedure is applied to limit a set of texture characteristics that provides a good classification with a small computational load. This procedure includes visual assessment of scatter plots, comparing values of texture features in different combinations. Candidate texture features that provide the best separation of classes are selected and others are discarded. The selection procedure also uses a set of training images to establish the set of texture features and its computation parameters, providing the smallest classification error. In other words, we constrain the texture features number by the demanded balance considering the SAR image level of details, computation time, and the optimal reliable class separation.

\subsection{Support vector machines}

The next step is the training of classifier (e.g., SVM) for classification of arrays with certain texture features as well as $\sigma^{\circ}$ values based on the results of manual classification. The SVM are supervised learning methods with associated learning algorithms that provide data classification. The basic SVM takes a set of input data (several "attributes", i.e., the features) and predicts the outputs (i.e., the class labels) for each given input, making it a non-probabilistic classifier. The support vector network maps the input vectors into a high dimensional feature space through nonlinear mapping. SVM finds a linear hyperplane separating objects into classes by the most widely clear gap between the nearest training data points of any class. An optimal hyperplane is defined as the linear decision function with maximal margin between the vectors in this higher dimensional space. When the maximum margin is found, only points which lie closest to the hyperplane have weights $>0$. These points determine this margin and are called support vectors (Cortes and Vapnik, 1995).

SVM performs a nonlinear classification using the kernel trick. The kernel function may transform the data into a higher dimensional space to make this nonlinearly separation possible when the relation between class labels and attributes is nonlinear. A common choice is a Gaussian kernel. In our study we have used the radial basis function kernel (RBF kernel), which is found to work well in a wide variety of applications.

The scikit-learn open source was used to implement the SVM classification method (http://scikit-learn.org/stable/ index.html). SVM models implementation in scikit-learn is based on LIBSVM. Basically, SVM trains the model using low-level method and can only solve binary classification problems. In the case of multi-class classification, LIBSVM implements the "one-against-one" technique by fitting all binary sub-classifiers and finding the correct class by a voting mechanism. The effectiveness of SVM training depends on the selection of kernel, the kernel's parameters $(\gamma)$, and margin parameter $C$. The software provides a simple tool to check a grid of parameters obtaining cross-validation accuracy for each parameter setting: the parameters with the highest cross-validation accuracy are returned (Hsu et al., 2003). The SVM parameters in our case were $\gamma=0.1$ and $C=1$.

The calculated texture features and $\sigma^{\circ}$ values corresponding to the manually identified classes on several preprocessed RS2 images were used as input data for training the SVM classifier. After completing the training procedure the resulting SVM is applied for automatic sea ice classification to divide the RS2 scene into the predefined classes.

\subsection{Validation}

The final step includes validation of the classification results using manually drawn ice charts. Validation of Arctic sea ice classification results is a challenging task since sea ice is a very inhomogeneous medium and validation data on ice classification are difficult to obtain. As a substitute our sea ice classification results have been compared with manual sea ice charts produced by the operational ice service at the Norwegian Meteorological Institute (MET Norway, http://polarview.met.no/). MET Norway produces ice charts every workday using the following data sources: highresolution SAR images, low-resolution microwave SSM/I and SSMIS data (DMSP), MODIS images (Terra and Aqua), and AVHRR data from NOAA. In our comparison MET Norway ice charts are assumed to represent "true" classification and the confusion matrix was calculated for accuracy evaluation of our algorithm results.

After completing the algorithm training, the fully automated image classification includes only three of the above mentioned steps: pre-processing (Sect. 3.1 and 3.2), texture feature retrieval (Sect. 3.4), and application of the automatic classifier (SVM).

The initial size of the full-resolution RS2 SCW image is about $10000 \times 10000$ pixels. We downscale the original image by averaging to $5000 \times 5000$ pixels to increase the computational efficiency and decrease the influence of speckle 
noise. The image size is further reduced during the computation of the texture features by using a sliding window with 16 pixel step size (the detailed parameters are described in Sect. 4.2). The image size of the final product is about $330 \times 330$ pixels with $1600 \mathrm{~m}$ pixel spacing. This reduction in resolution significantly increases the processing speed and allows computing a classification results in less than $15 \mathrm{~min}$.

Pre-processing of RS2 data was performed utilizing the open-source Python toolbox NANSAT (Korosov et al., 2015), (https://github.com/nansencenter/nansat/wiki). The texture extraction algorithm was created in the Python programming language.

\section{Results}

To illustrate the algorithm performance the automatic SVM classification was applied to the RS2 scene shown in Fig. 2. The example scene was acquired on 28 November 2011 over the western part of Svalbard in Fram Strait. Figures 2 and 3 show both $\mathrm{HH}$ and HV polarizations before and after corresponding corrections described in Sect. 2: compensation of incidence angle effects for $\mathrm{HH}$ (Fig. 3a) and noise reduction for HV (Fig. 3b). The image contains several ice types, open water under different wind conditions, and land. The open water area is located on the right-hand side of the image and the ice-covered area in the upper-left corner. The sea ice area includes a marginal ice zone with bright broken up ice. The ice-covered areas and the rough OW areas appear both bright in $\mathrm{HH}$ and are therefore difficult to distinguish. Including HV, however, provides additional information since OW areas on this image appear generally darker than sea ice in HV. This is one of the major dual-polarization advantages and can be seen in the lower right part of the example image (Fig. 2).

\subsection{Correction for incidence angle and thermal noise}

The linear trend coefficient used for backscatter angular dependence correction of $\mathrm{HH}$ was estimated to be $-0.298 \mathrm{~dB} / 1^{\circ}$ and allowed normalization of $\sigma^{\circ}$ to the incidence angle $35^{\circ}$ as shown on Fig. 3a and c. The application of our noise correction procedure for $\mathrm{HV}$ reduces significantly thermal noise and gets rid of vertical striping as shown in Fig. 3b, d.

\subsection{Texture feature calculation}

As part of the algorithm development texture features were calculated based on different parameter settings. Visual examination of mean values of several texture features (Fig. 4a, b) suggested the optimal combination of the sliding window, moving step, and distance between neighboring pixels, which provides better separation of the ice-water classes compared to other combinations of window sizes with different texture parameters. A set of texture characteristics was selected analyzing variations in mean values of the textu- ral characteristics of defined classes calculated with several combinations of obtained parameters (Fig. 4c, d). The largest change of distance between mean values of texture features of different classes on Fig. 4d defines the best option for the potential classification. Finally, together with visual inspection of the texture images (some examples are given on Fig. 5a-f) of the a priori known most problematic classification cases on the SAR images used for training, the set of texture characteristics are defined. The best results were achieved using the following parameter set: number of gray levels $(K=32)$, co-occurrence distance $(d=8)$, sliding window size $(w=64 \times 64)$, and moving step of the sliding window $(s=16)$. Using the following texture features for the two channels provided the best test results: for $\mathrm{HH}$ channel the energy, inertia, cluster prominence, entropy, third statistical moment of brightness, backscatter, and standard deviation were calculated; for HV channel the energy, correlation, homogeneity, entropy, and backscatter were calculated. Including more texture features for both channels was tested but found not to improve the information content. The calculation parameters were found experimentally to give a good compromise between speckle noise reduction, preservation of details, and correct classification results (methodology description in Zakhvatkina et al., 2013).

Texture characteristics provide a more complete delineation of surface parameters in addition to the raw backscatter signal, and increase the ability for ice and water separation. The scatter plots in Fig. 5g, h show the values of two different texture features plotted against each other and illustrate the usefulness of texture features for discrimination between defined classes.

\subsection{Manual versus automatic classification}

As described in Sect. 3 several SAR images were classified manually as part of the training procedure for the automatic algorithm. Comparing the manual classification from sea ice expert analysis with the algorithm results (Fig. 6) reveals a general high level of correspondence and illustrates the capability of the automatic approach. Detailed observation of the classification results show that most misclassifications are observed near land and in the MIZ. Figure $6 \mathrm{~b}$ shows small features inside ice-covered zone (blue dots) that were misclassified as OW.

\subsection{Validation}

Validation of the algorithm results has been performed using 2705 RS2 images taken over our area of interest in the period 1 January 2013 until 25 October 2015. For each RS2 image an error matrix based on pixel-by-pixel difference between algorithm result and MET Norway chart has been calculated. OW and sea ice correspondence as well as an overall accuracy were obtained for each RS2 image classification result and averaged accuracies have been calculated for 


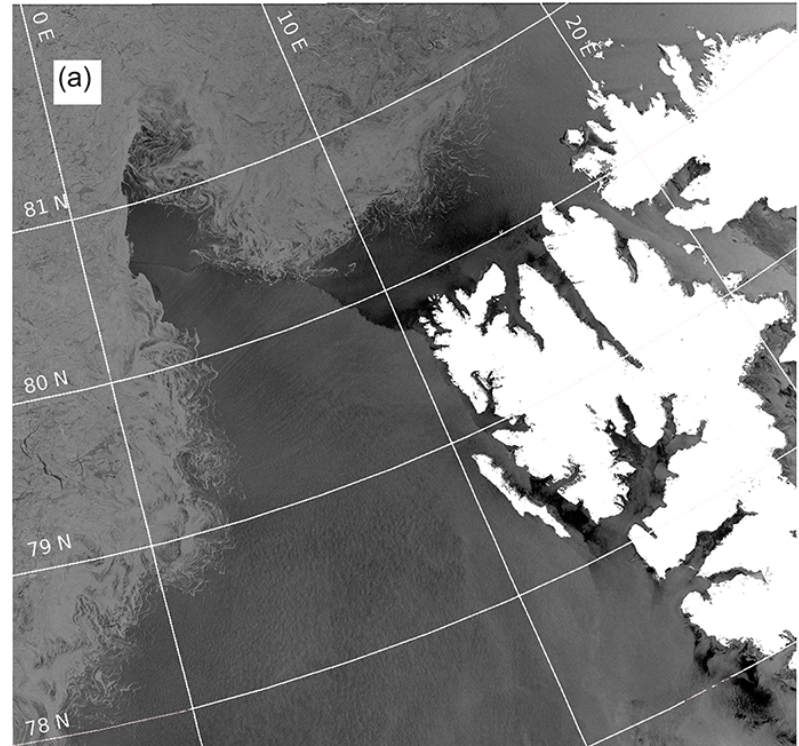

(c)

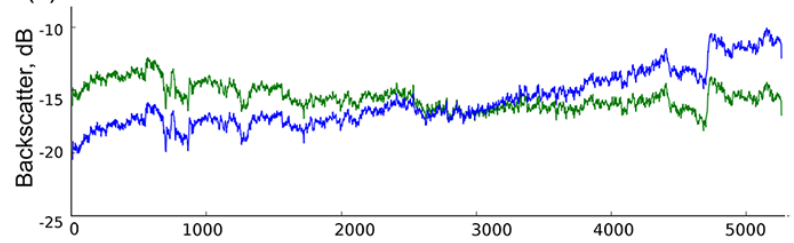

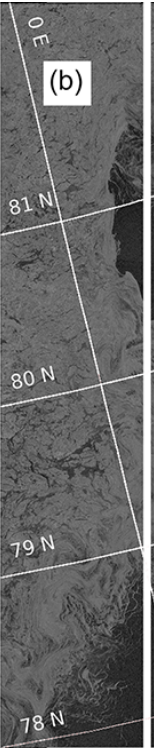
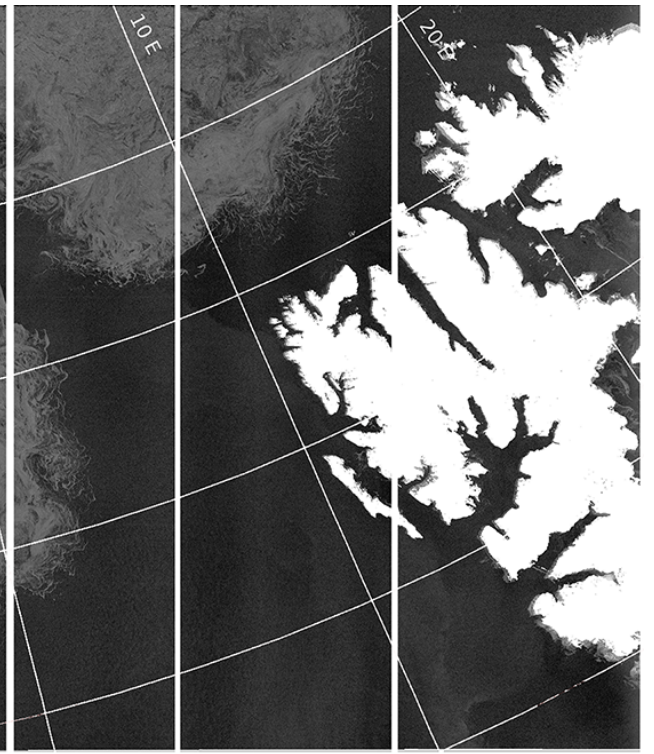

(d)

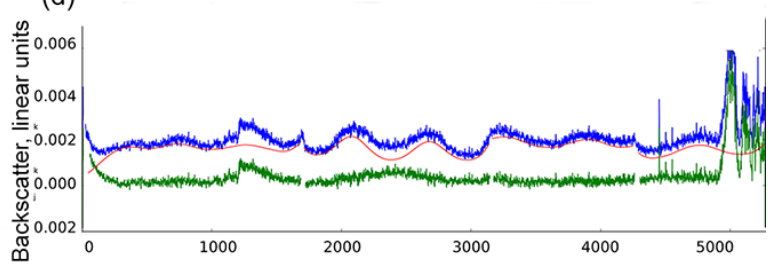

Figure 3. RS2 SCW dual-polarization image taken over Fram Strait on 28 November 2011, including pre-processing. (a) Calibrated image after correction of $\sigma^{\circ}$ at $35^{\circ}$ incidence angle using the predefined coefficient for sea ice of $-0.298 \mathrm{~dB} / 1^{\circ}$. (b) Noise-corrected image: beam boundaries are visible due to differences in noise levels between adjacent beams. (c) $\sigma^{\circ}$ curves of SAR image across the entire swath: original image (blue) and after angular correction (green). (d) $\sigma^{\circ}$ curves of SAR image along the whole swath. The blue curve shows $\sigma^{\circ}$ value profile of the raw HV channel image in range direction, the red curve depicts the noise floor level, and the green curve is the result of subtraction.

each month. The impact of each class on the classification error has been estimated and the respective monthly averaged errors were computed. The averaged overall accuracies including standard deviation and errors in ice and water classification for each month are given in Table 2. In addition, the monthly accuracies are presented as a graph in Fig. 7. The monthly averaged overall accuracies show lower values during summer months (Fig. 7 - from May to October) and higher values during winter. The average total classification accuracy for all 2705 scenes is $91 \pm 4 \%$.

Figure 8 shows an example of the validation process. The RS2 HH image is shown in Fig. 8a, the result of our SVM classification in Fig. 8c, and the MET Norway sea ice chart in Fig. 8b. To compare the algorithm result with the manually derived ice charts, both products are reclassified into ice and water (Fig. 8d and e). The error matrix is represented as an image (Fig. 8f) with the following three classes: no difference, sea ice error (MET Norway: sea ice, OW in our results), and OW error (MET Norway: OW, sea ice in our results).

\section{Discussion}

\subsection{Significance of incidence angle variations and thermal noise reduction}

Water areas have a very large range of brightness depending on wind speed. At higher wind speeds the contrast between open water and first- and multi-year ice is reduced, which gives an ambiguity between these classes. The dependence of backscatter on incidence angle is well known (Shokr, 2009) and is significantly higher for open water than for sea ice. The correction factor for the incidence angle is therefore very different for ice and water. The coefficients for the angular dependence of water-covered areas are significantly influenced by wind conditions - with stronger wind intensity grows faster. Our observations show that angular dependence of sea ice is more stable regardless of wind or other conditions (Fig. 3). Since the surface type is not known a priori we have to choose which angular correction to apply and the preference is given to the more reliable sea ice angular correction. However, the total compensation is impossible as the backscatter dependence on the incidence angle varies for dif- 
(a)

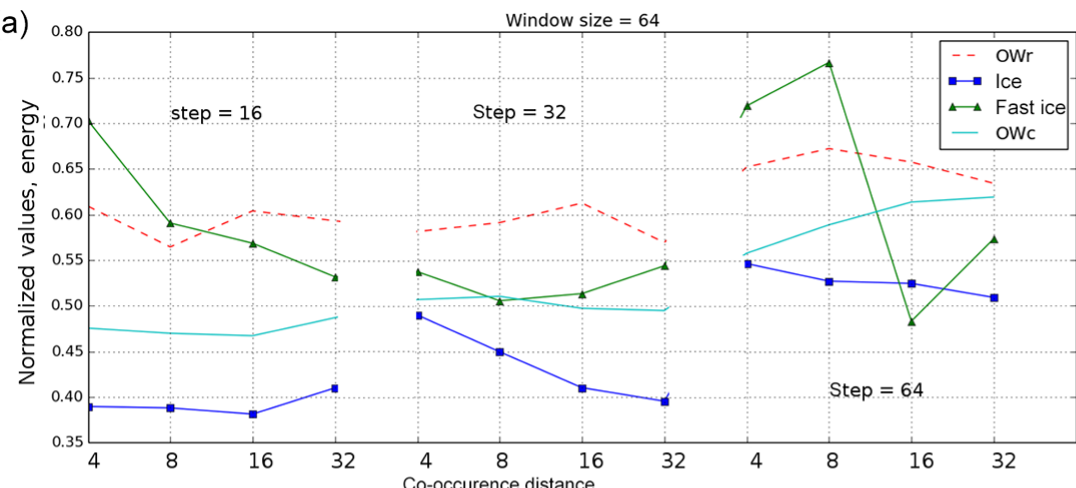

(b)

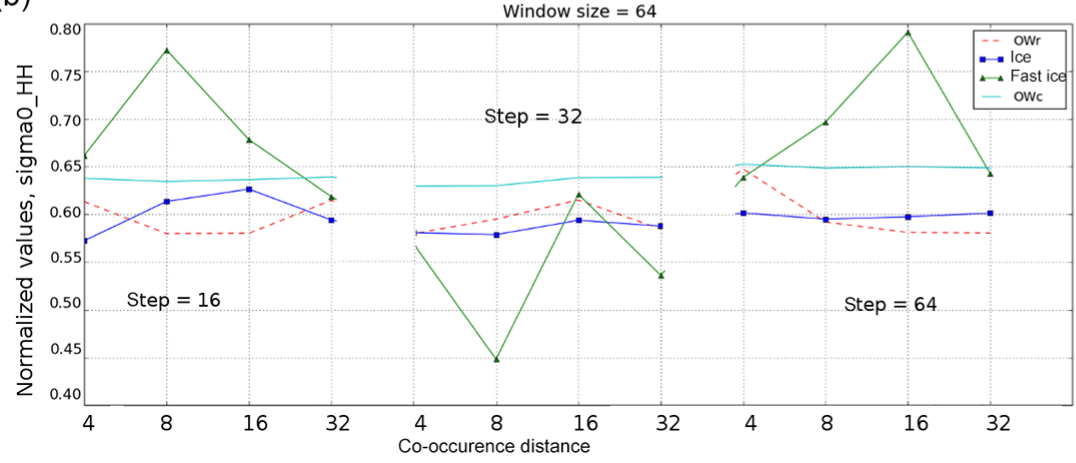

(c)

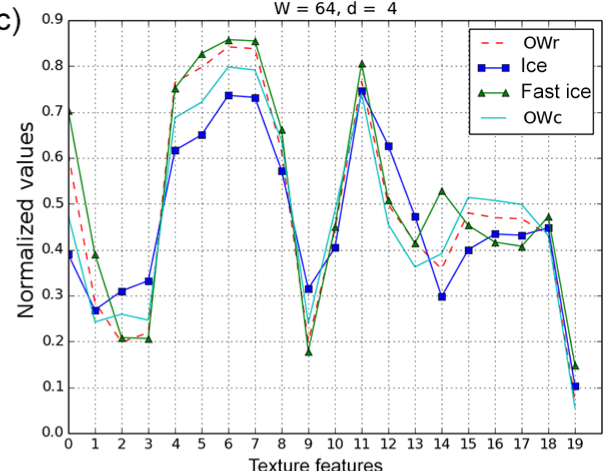

(d)

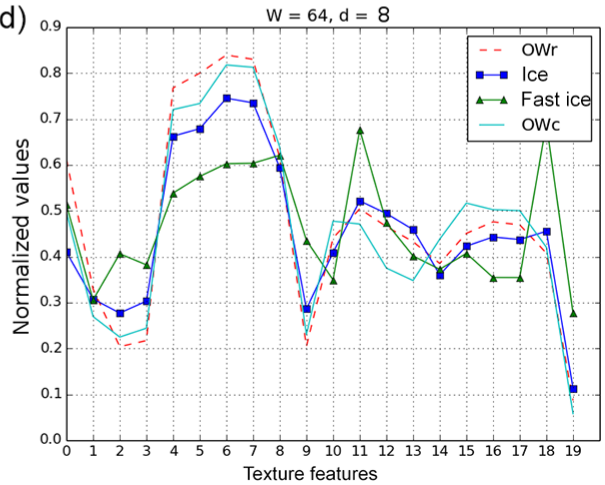

Figure 4. Normalized mean values of texture characteristics for calm open water $(\mathrm{OWc})$, rough open water $(\mathrm{OWr})$, ice, and fast ice, calculated in window size $64 \times 64$ pixels: (a) energy and (b) $\sigma^{\circ}$ of $\mathrm{HH}$ with different co-occurrence distances for several moving step variations. Set of texture features are calculated with found above parameters: (c) $d=4$ and step $=16 ;$ (d) $d=8$ and step $=16$ pixels $(0-$ energy, $1-$ correlation, 2 - inertia or contrast, 3 - cluster prominence, 4 - homogeneity, 5 - entropy, 6 - third central statistical moment of brightness, 7 - fourth central statistical moment of brightness, 8 - average sea ice backscatter, 9 - standard deviation of brightness for $\mathrm{HH}$ ). The range from 10 to 19 indicates the same texture features calculated for HV. The calculations were made for several images used for training.

Table 2. Monthly averaged accuracies of the automatic ice charts compared to MET Norway ice charts (results given in \%).

\begin{tabular}{|c|c|c|c|c|c|c|c|c|c|c|c|c|c|c|c|c|c|}
\hline \multicolumn{6}{|c|}{2013} & \multicolumn{6}{|c|}{2014} & \multicolumn{6}{|c|}{2015} \\
\hline Months & Images & Ov acc & SD & OW err & Ice err & Months & Images & Ov acc & SD & OW err & Ice err & Months & Images & Ov acc & SD & OW err & Ice err \\
\hline Jan & 72 & 91.52 & 5.43 & 3.99 & 4.50 & Jan & 97 & 91.89 & 4.70 & 2.52 & 5.59 & Jan & 51 & 94.84 & 3.10 & 1.28 & 3.88 \\
\hline Feb & 70 & 91.05 & 4.54 & 2.66 & 6.30 & Feb & 93 & 92.11 & 5.05 & 3.37 & 4.52 & Feb & 33 & 94.47 & 4.05 & 2.33 & 3.86 \\
\hline Mar & 106 & 91.21 & 4.71 & 1.20 & 7.59 & Mar & 110 & 92.20 & 3.45 & 2.83 & 4.98 & Mar & 73 & 94.36 & 4.40 & 1.67 & 3.82 \\
\hline Apr & 110 & 92.03 & 4.57 & 0.95 & 7.02 & Apr & 130 & 93.34 & 3.40 & 1.30 & 5.36 & Apr & 54 & 94.86 & 4.36 & 1.47 & 3.83 \\
\hline May & 111 & 88.60 & 7.96 & 0.88 & 10.52 & May & 137 & 92.80 & 4.77 & 1.00 & 6.20 & May & 63 & 95.05 & 3.21 & 0.72 & 3.81 \\
\hline Jun & 98 & 87.64 & 7.58 & 1.59 & 10.76 & Jun & 93 & 89.98 & 5.78 & 1.54 & 8.48 & Jun & 67 & 84.73 & 14.09 & 0.69 & 3.80 \\
\hline Jul & 83 & 89.73 & 8.01 & 2.72 & 7.54 & Jul & 95 & 86.82 & 9.89 & 1.98 & 11.20 & Jul & 47 & 74.49 & 21.61 & 1.73 & 3.81 \\
\hline Aug & 85 & 94.36 & 3.10 & 2.96 & 2.68 & Aug & 88 & 88.39 & 10.87 & 1.87 & 9.74 & Aug & 47 & 86.65 & 12.25 & 2.64 & 3.85 \\
\hline Sep & 93 & 95.88 & 2.02 & 2.47 & 1.65 & Sep & 97 & 87.55 & 17.56 & 8.24 & 4.21 & Sep & 43 & 94.83 & 3.87 & 3.36 & 3.78 \\
\hline Okt & 72 & 94.53 & 2.99 & 3.98 & 1.49 & Okt & 78 & 94.89 & 3.15 & 1.87 & 3.24 & Okt & 27 & 94.69 & 4.16 & 4.58 & 3.78 \\
\hline Nov & 84 & 92.00 & 4.77 & 5.10 & 2.90 & Nov & 47 & 94.58 & 2.84 & 2.38 & 3.04 & Nov & & & & & \\
\hline Dec & 97 & 90.93 & 6.63 & 3.18 & 5.88 & Dec & 54 & 92.94 & 7.99 & 3.45 & 3.61 & Dec & & & & & \\
\hline
\end{tabular}
chart.

ferent ice types (Mäkynen et al., 2002) and water areas in the scene. The radiometric corrections during calibration process are just a first-order approximation; nevertheless, the advantages of performing the angular correction are greater than the disadvantages (Moen et al., 2015). With regards to thermal noise correction we found that sometimes not all visible noise floor artifacts inside beams can be completely removed and these residuals may cause classification errors.

\subsection{Number of texture features vs. efficiency}

In addition to the eigth extracted texture features we characterize the surface by values of $\sigma^{\circ}$ averaged within the sliding window and a value of standard deviations. Given that we have two channels (HH and HV) the number of parameters grows up to 20 and some of them are strongly intercorrelated (Shokr, 1991; Albregtsen, 2008). High correlation be- 

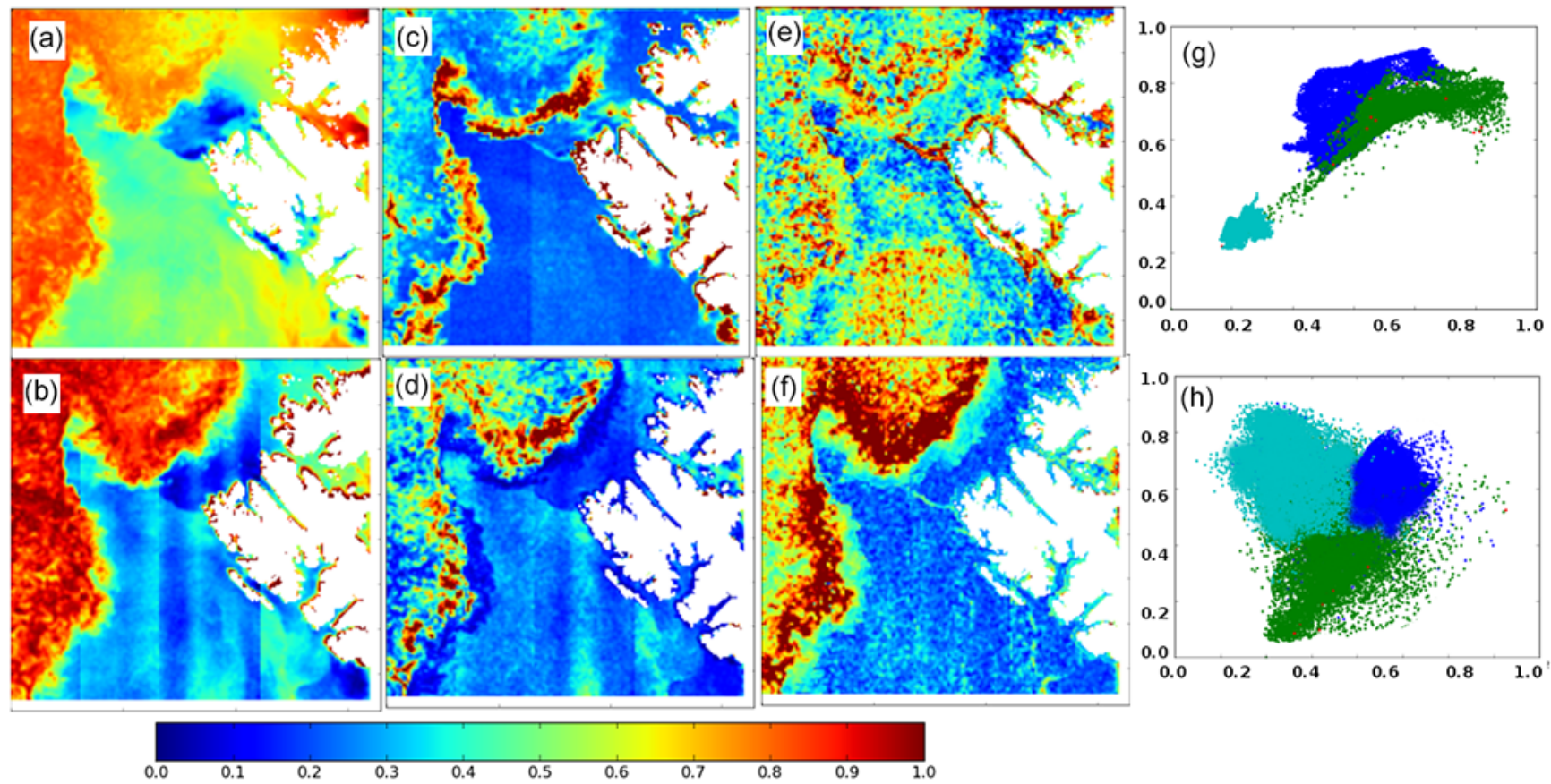

Figure 5. Texture features calculated for RS2 SCW scene, 28 November 2011, in the Fram Strait. (a) Backscatter of HH polarization; (b) backscatter of HV polarization; (c) inertia of HH polarization; (d) energy of HV polarization; (e) correlation of HH polarization; (f) correlation of HV polarization. The scatter plots show how a couple of textural features calculated from RS2 images, shown in Fig. 1, can be used to classify ice (green), rough OW (blue), and calm OW (cyan). (g) $\sigma^{\circ}$ of HV vs. $\sigma^{\circ}$ of HH. (h) Energy of HH vs. correlation of HV.

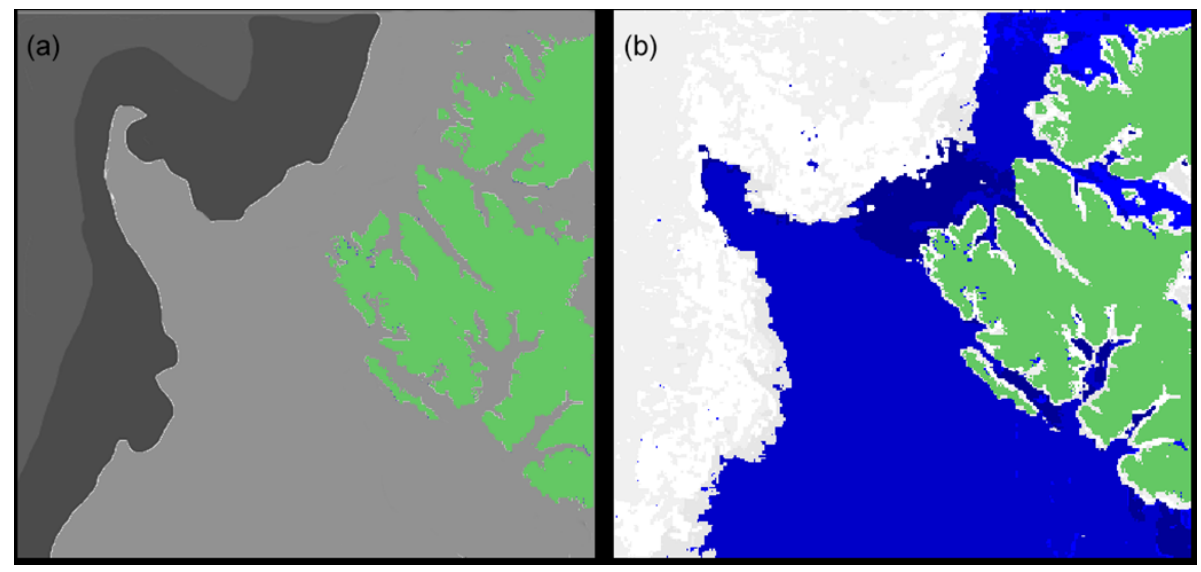

Figure 6. OW and sea ice classification of RS2 SCW image shown in Fig. 2. (a) Manual classification based on sea ice expert analysis to delineate sea ice (in the MIZ and general sea ice cover) and open water (calm and rough open water): dark gray is sea ice; very dark gray is marginal ice zone; light gray is OW; green is land. (b) Automatic SVM classification result: white is sea ice; dark blue is calm OW; blue is OW; green is land.

tween two textural characteristics shows that they have similar properties, and hence it makes no sense to use both features. In case of low correlation both features will contribute to the improvement of the classification accuracy (Clausi, 2002). The similarity can explain the misclassifications and in fact this is part of the motivation to reduce dimensionality. If we include too few texture features to the classifier then the informationally poor features have to be compensated by using complicated discrimination function and can lead to increased classification confusion. In contrast, if all texture features are used by the classifier, some classes can be underestimated or overestimated and the discrimination for many classes may lead to higher classification errors. 


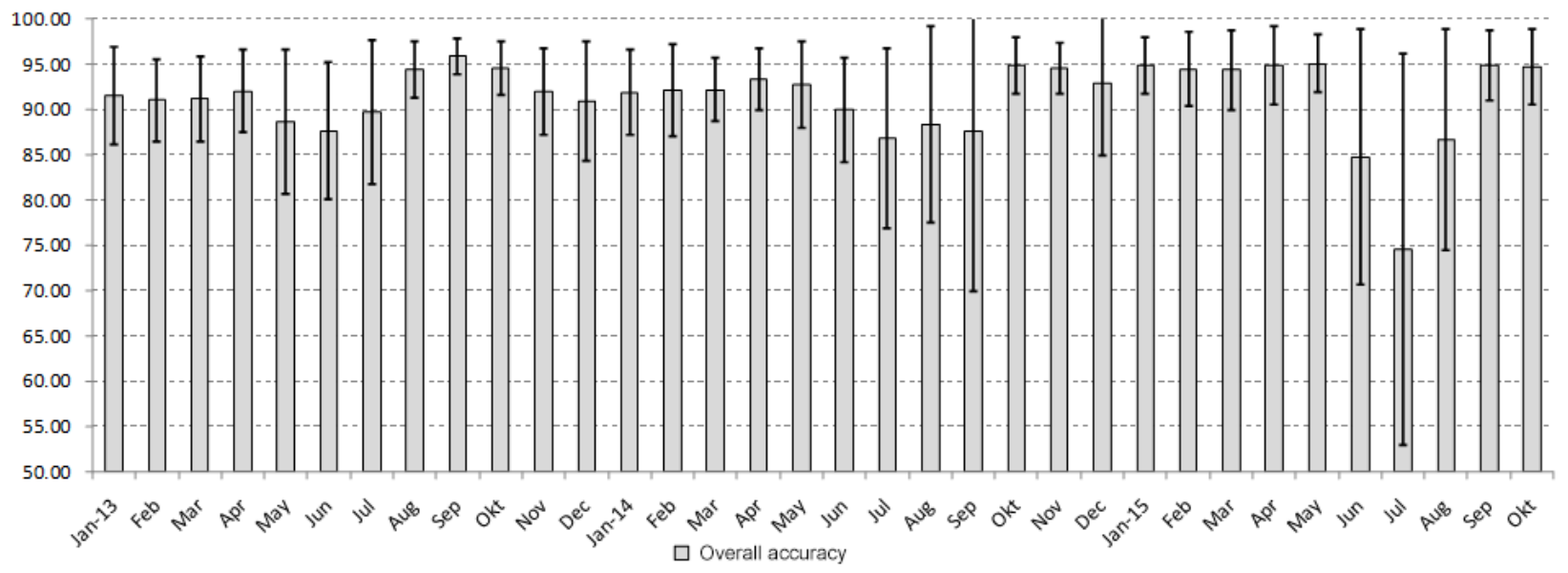

Figure 7. Monthly accuracy and standard deviation of SVM classification of RS2 images assuming that MET Norway operational ice charts are correct.

Sea ice in the upper part of Fig. 5a could not be distinguished from rough open water (upper right). However, Fig. 5b shows reliable detection of sea ice-covered area (left side). Calm open water can be easily recognized in Fig. 5c and d (dark blue areas). In both figures, the heterogeneous sea ice area can be clearly distinguished from the open water zone. The latter consist of very close ice floes and/or broken ice. Some other ice-covered area can be incorrectly defined as open water. Figure 5e adds more useful information about open water location (blue colored area). The scatter plot on Fig. $5 \mathrm{~g}, \mathrm{~h}$ represents advantage of texture feature application for discrimination between the sea ice and two classes of open water using both polarizations, where sea ice (green) can be clearly seen as standing separately from OW (blue). The scatter plot in Fig. 5h demonstrates how different texture characteristics, e.g., energy versus correlation, of different polarizations can add useful information for detection. The examples in Fig. 5e and $\mathrm{f}$ show that the same texture feature calculated for one polarization can be used in applications to obtain well-delineated class; otherwise for other polarization it demonstrates the poor separation between classes.

\subsection{Sources of errors}

The MET Norway manual products and our algorithm results show generally a good consistency. However, differences typically appear at the ice-water boundary and inside ice-covered areas, where leads or channels on the SAR image are not delineated on the MET Norway ice charts. Some differences are also found in the coastal zones, where narrow ice zones near the coast are wrongly shown in our results or fast ice is wrongly classified as OW by our algorithm. This misclassification can be explained by appearance of fast ice and calm open water on a SAR image and its similarity in the low backscatter. For this case the polarization difference in backscatter between $\mathrm{HH}$ and $\mathrm{HV}$ bands (cross-polarization ratio) could be included for further improvement (Sandven, 2008; Dierking and Pedersen, 2012; Moen et al., 2013). More significant classification errors can be found in the MIZ.

Detecting typical backscatter ranges and textural structures for different sea ice types and water areas with different roughness stages is extremely difficult due to the high dynamic and variable nature of sea ice and wind speed impact. In particular, different structures on the water affected by wind and currents and visually detected on the SAR images (e.g., stripes, eddies) may cause wrong sea ice classification.

Residual HV noise effects (after correction) along the ScanSAR image beam boundaries and signal variations inside the separate beams due to instrumental artifacts (Fig. 5b, d) can have an uncorrected effect on the texture feature analysis and may cause classification errors. These residual noise effects are not visible in the ice-covered areas, but rough open water on high incidence angle close to the beam boundaries may be erroneously classified as sea ice.

The backscatter signal of melting ice becomes similar to open water and imposes limitations for the classification of RS2 images for the summer season.

We assume that our automatic algorithm classifies SAR images more reliable as than represented by the provided accuracy $(91 \%)$, and this inconsistency may occur for the following reasons:

1. The MET Norway ice charts have a lower resolution than our automatic ice charts making an absolute accurate estimation of the ice conditions in the each SAR images and detailed comparison impossible.

2. The classes obtained by MET Norway are not consistent with the simple ice-water classification provided by the algorithm. In the comparison, we reclassify the MET Norway ice chart into ice and open water. Here, areas with ice concentrations $\leq 10 \%$ are regarded as open wa- 

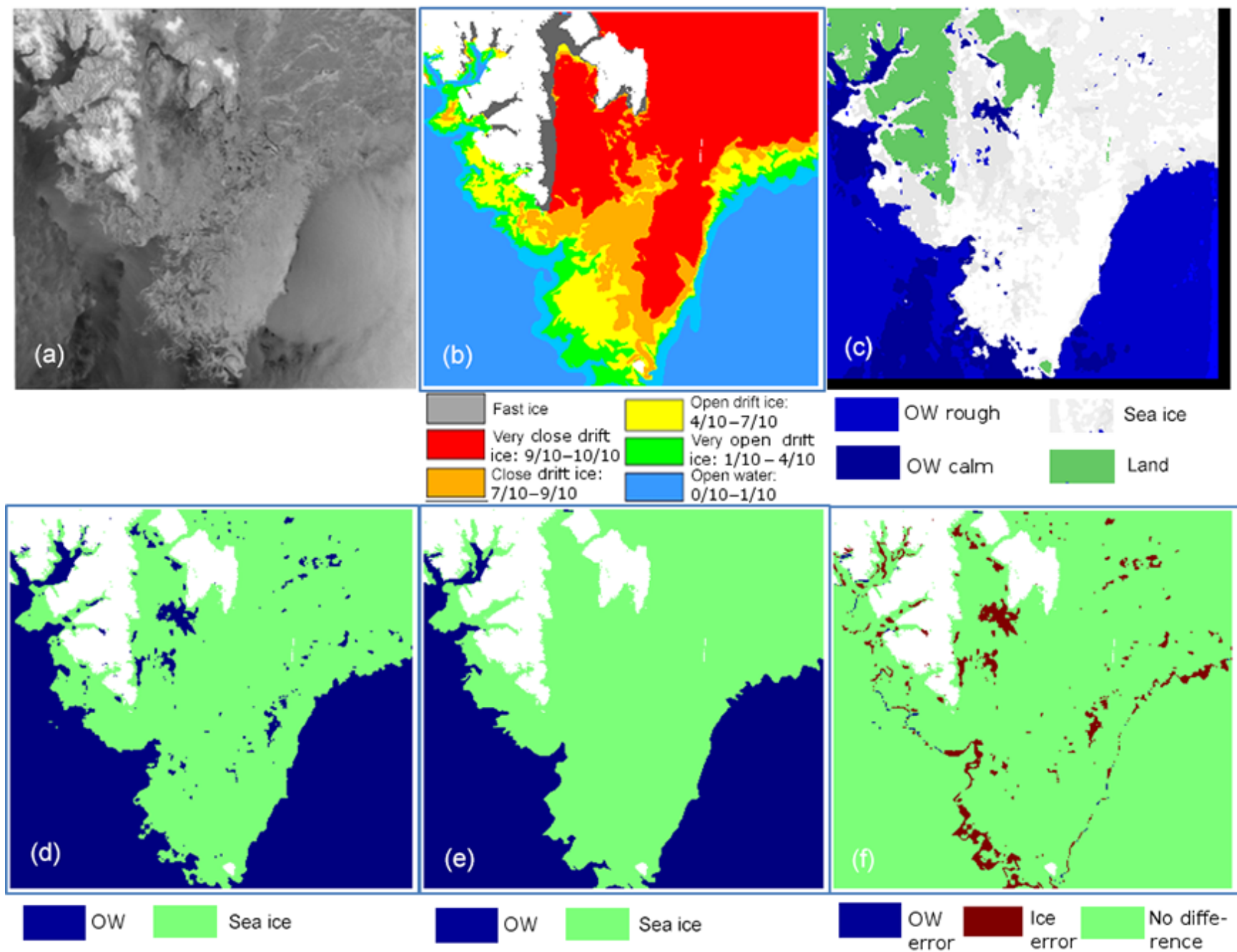

Figure 8. Validation procedure of automatic classification results compared to MET Norway ice charts. (a) Original RS2 SCW SAR image (HH polarization), taken over the southern part of Svalbard on 14 March 2013. (b) Collocated subset of manual ice concentration chart, provided by the Norwegian Ice Service (met.no) for the same day. (c) Result of the SVM classification. (d) Result of the SVM classification with delineation of two classes: water and sea ice. (e) Ice chart of MET Norway reclassified into two classes: open water (ice concentration from 0 to $10 \%$ ) and sea ice (ice concentration from 10 to $100 \%$ ). (f) The difference of recalculated MET Norway chart and classification result represents the error matrix as "image": no difference, sea ice error (sea ice in MET Norway, OW in our results), and OW error (OW in MET Norway, sea ice in our results). Overall accuracy is $95.78 \%$, OW error is $0.19 \%$, and ice error is $4.03 \%$.

ter. This assumption appears to be the subjective error factor during the validation process and finally reduces the accuracy.

3. MET Norway provides manual ice charts for every working day, but not for weekends and holidays. This might cause a difference in timing up to several days. Manual and automatic ice charts of the same day might also not be based on images taken at the same time of the day. Fram Strait is a very dynamic region and the sea ice situation can significantly change over time periods of several hours.

\section{Conclusion}

We have proposed an automated OW-ice cover classification of RADARSAT-2 SAR ScanSAR Wide Beam mode data acquired over Fram Strait for varying wind speeds and sea ice conditions. The classification uses backscatter and texture features together in a SVM approach. The intensity contrast between $\mathrm{HH}$ and $\mathrm{HV}$ polarization of open water increases at higher wind speeds, and open water is distinguished more reliably on dual-polarized RS2 data.

Previous studies of ENVISAT ASAR HH data in wide swath mode showed a similar backscatter dependence on incidence angle (Zakhvatkina et al., 2013), and the same tech- 
nique was applied for the HH band of RS2 SCW images. The ScanSAR image swath consists of different combinations of four physical beams and there are well-known technical features caused by a wave-like modulation of the image intensity in range direction throughout the entire image in the sub-swaths and their edges of HV band (Romeiser et al., 2013). Although the techniques for compensating the effect in the SAR processor have been developed and applied, some ScanSAR images still show residual effects. To improve utilization of such images we have carried out a procedure of $\mathrm{HV}$ band noise reduction that is applied as a preprocessing tool. By computing texture features with sliding window size of $64 \times 64$ pixels and number of quantized gray levels amounting to 32 , we classified more that 2700 SAR images for the period from January 2013 to October 2015. Validation of the classification was done by comparing with ice charts produced by MET Norway. The texture features were used as input to SVM classification. The results show that open water and ice are discriminated with an accuracy of $91 \%$.

The automated SVM-based algorithm has been adopted for operational decoding the ice edge, and it will also be extended and improved for sea ice type classification. With Sentinel-1A/B as the main satellite SAR system in the coming years, the next step will be to adapt the classification algorithm to Sentinel-1 data (Korosov, 2016). The amount of SAR data available for sea ice monitoring will increase significantly in the coming years. Efficient utilization of these data will require further efforts to develop automated algorithms which can be used in operational ice services.

\section{Data availability}

The RADARSAT- 2 data used in this study are not publicly accessible because RADARSAT- 2 is a commercial satellite. We obtained the data used in the study as MyOcean users under a special contingency agreement between ESA and MDA GSI.

Acknowledgements. The work has been supported by the EU projects MyOcean (grant agreement no. 218812), SIDARUS (grant agreement no. 262922), MAIRES (grant agreement no. 263165), Research Council of Norway (contract 196214), Norwegian Space Center (JOP.01.11.2), and EuRuCAS (grant agreement no. 295068), SONARC project under Research Council of Norway (contract no. 243608), and Russian Foundation for Basic Research (RFBR) project SONARC (grant agreement no. 15-55-20002). We acknowledge the MDA for providing RADARSAT-2 data through SIDARUS project. Authors would thank Nick Hughes from Norwegian Meteorological Institute for providing high-resolution ice charts for validation of the classification method.

Edited by: C. Duguay

Reviewed by: W. Dierking and two anonymous referees

\section{References}

Albregtsen, F.: Statistical Texture Measures Computed from Gray Level Coocurrence Matrices, Image Processing Laboratory Department of Informatics University of Oslo, 5 November 2008.

Bogdanov, A. V., Sandven, S., Johannessen, O. M., Alexandrov, V. Y., and Bobylev, L. P.: Multisensor approach to automated classification of sea ice image data, IEEE T. Geosci. Remote, 43, 1648-1664, 2005.

Clausi, D. A.: An analysis of co-occurrence texture statistics as a function of grey level quantization, Can. J. Remote Sens., 28, 45-62, 2002.

Clausi, D. A., Qin, A. K., Chowdhury, M. S., Yu, P., and Maillard, P.: MAGIC: MAp-Guided Ice Classification System, Can. J. Remote Sens., 36, suppl. 1, S13-S25, 2010.

Cortes, C. and Vapnik, V.: Support-Vector Networks, Mach. Learn., 20, 273-297, 1995.

Dierking, W.: Mapping of different sea ice regimes using images from Sentinel-1 and ALOS synthetic aperture radar, IEEE T. Geosci. Remote, 48, 1045-105, 2010.

Dierking, W.: Sea ice monitoring by synthetic aperture radar, Oceanography, 26, 100-111, 2013.

Dierking, W. and Pedersen, L.: Monitoring sea ice using ENVISAT ASAR - A new era starting 10 years ago, Proc. IEEE Int. Geosci. Remote SE (IGARSS2012), 1852-1855, 2012.

Dokken, S. T., Markus, P. W. T., Askne, J., and Bjork, G.: ERS SAR characterization of coastal polynyas in the Arctic and comparison with SSM/I and numerical model investigations, Remote Sens. Environ., 80, 321-335, 2002.

Geldsetzer, T. and Yackel, J. J.: Sea ice type and open water discrimination using dual co-polarized C-band SAR, Can. J. Remote Sens., 35, 73-84, 2009.

Gill, R. S.: SAR Ice Classification Using Fuzzy Screening Method, in: Proc. Workshop on Applications of SAR Polarimetry and Polarimetric Interferometry (POLinSAR), 14-16 January 2003, Frascati, Italy, 2003.

Gill, J. P. S. and Yackel, J. J.: Evaluation of C-band SAR polarimetric parameters for discriminating of first-year sea ice types, Can. J. Remote Sens., 38, 306-323, 2012.

Haarpaintner, J. and Solbø, S.: Automatic ice-ocean discrimination in SAR imagery, Norut IT-report, Tech. Rep., 2007.

Haralick, R. M., Shanmugam, K. S., and Dinstein, I.: Textural features for image classification, IEEE T. Syst. Man. Cyb., 3, 610 621, 1973.

Hsu, C.-W., Chang, C.-C., and Lin, C.-J.: A practical guide to support vector classification, Initial version: 2003, available at: http://www.csie.ntu.edu.tw/ cjlin/papers/guide/guide. pdf, last access: 8 June 2016.

Jefferies, B.: Radarsat-2 - New Ice Information Products, Proc. of the 13th Meeting - International Ice Charting Working Group, 15-19 October 2012, Tromso, Norway, 2012.

Johannessen, O. M., Alexandrov, V., Frolov, Bobylev, L., Sandven, S., Miles, M., Pettersson, L., Kloster, K., Smirnov, V., Mironov, Y., and Babich, N.: Remote Sensing of Sea Ice in the Northern Sea Route: Studies and Applications, Chichester, UK, Springer-Praxis, available at: https://www.nersc.no/sites/www. nersc.no/files/front-matter.pdf, 2007.

Karvonen, J.: C-band sea ice SAR classification based on segmentwise edge features, in: Geoscience and Remote Sensing New 
Achievements, edited by: Imperatore, P. and Riccio, D., In- Tech, 129-146, 2010.

Karvonen, J.: Operational SAR-based sea ice drift monitoring over the Baltic Sea, Ocean Sci., 8, 473-483, 2012.

Karvonen, J., Simila, M., and Mäkynen, M.: Open Water Detection from Baltic Sea Ice Radarsat-1 SAR Imagery, IEEE T. Geosci. Remote Lett., 2, 275-279, 2005.

Korosov, A.: Very high resolution classification of Sentinel-1A data using segmentation and texture features, Proc. of European Space Agency Living Planet Symposium, 9-13 May 2016, Prague, Czech Republic, 2016.

Korosov, A., Hansen, M. W., and Yamakava, A.: Nansat - scientist friendly toolbox for processing satellite data, World Ocean Scientific Congress, 2-8 February 2015, Cochin, India, 2015.

Leigh, S., Zhijie, W., and Clausi, D. A.: Automated Ice-Water Classification Using Dual Polarization SAR Satellite Imagery, IEEE T. Geosci. Remote, 52, 5529-5539, 2014.

Maillard, P., Clausi, D. A., and Deng, H.: Map-guided sea ice segmentation and classification using SAR imagery and a MRF segmentation scheme, IEEE T. Geosci. Remote, 43, 2940-2951, 2005.

Mäkynen, M. P., Manninen, A. T., Similä, M. H., Karvonen, J. A., and Hallikainen, M. T.: Incidence angle dependence of the statistical properties of C-band $\mathrm{HH}$-polarization backscattering signatures of the Baltic Sea ice, IEEE T. Geosci. Remote, 40, 2593 2605, 2002.

Moen, M.-A. N., Doulgeris, A. P., Anfinsen, S. N., Renner, A. H. H., Hughes, N., Gerland, S., and Eltoft, T.: Comparison of feature based segmentation of full polarimetric SAR satellite sea ice images with manually drawn ice charts, The Cryosphere, 7, 1693-1705, doi:10.5194/tc-7-1693-2013, 2013.

Moen, M.-A. N., Anfinsen, S. N., Doulgeris, A. P., Renner, A. H. H., and Gerland, S.: Assessing polarimetric SAR sea-ice classifications using consecutive day images, Ann. Glaciol., 56, 285-294, 2015.

Ochilov, S. and Clausi, D. A.: Operational SAR sea-ice image classification, IEEE T. Geosci. Remote, 50, 4397-4408, 2012.

RADARSAT-2 product description: MacDonald Dettwiler and Associates (MDA), Tech. Rep. Issue 1/8, available at: http://gs.mdacorporation.com/includes/documents/ RN-SP-52-1238RS-2ProductDescription1-8_15APR2011.pdf (last access: 1 July 2014), 15 April 2011.
Romeiser, R., Horstmann, J., Caruso, M. J., and Graber, H. C.: A descalloping post-processor for ScanSAR images of ocean scenes, IEEE T. Geosci. Remote, 51, 3259-3272, 2013.

Sandven, S., Kloster, K., Alexandrov, V., Piotrovskaya, N., and Zakhvatkina, N.: Sea ice classification using ASAR Alternating Polarisation images, SeaSAR 2008, 21-24 January 2008, Oslo, Norway, 2008.

Sandven, S., Alexandrov, V., Zakhvatkina, N., and Babiker, M.: Sea ice classification using RADARSAT-2 Dual Polarisation data, SeaSAR 2012, the 4th International Workshop on Advances in SAR Oceanography, 18-22 June 2012, Tromso, Norway, 2012.

Shokr, M. E.: Evaluation of Second-Order Texture Parameters for Sea Ice Classification from Radar Images, J. Geophys. Res., 96, 10625-10640, 1991.

Shokr, M. E.: Compilation of a radar backscatter database of sea ice types and open water using operational analysis of heterogeneous ice regimes, Can. J. Remote Sens., 35, 369-384, 2009.

Soh, L. K. and Tsatsoulis, C.: Texture analysis of SAR sea ice imagery using gray level co-occurrence matrices, IEEE T. Geosci. Remote, 37, 780-795, 1999.

Soh, L. K., Tsatsoulis, C., Gineris, D., and Bertoia, C.: ARKTOS: An intelligent system for SAR sea ice image classification, IEEE T. Geosci. Remote, 42, 229-248, 2004.

Vinje, T. and Finnekåsa, Ø.: The ice transport through the Fram Strait, Norsk Polar institutt Skriffer, 186, 39, 1986.

Yu, Q. and Clausi, D. A.: IRGS: Image segmentation using edge penalties and region growing, IEEE T. Pattern Anal., 30, 21262139, 2008.

Yu, P., Qin, A. K., and Clausi, D. A.: Feature extraction of dual-pol SAR imagery for sea ice image segmentation, Can. J. Remote Sens., 38, 352-366, 2012.

Zakhvatkina, N., Alexandrov, V., Johannessen, O. M., Sandven, S., and Frolov, I.: Classification of sea ice types in ENVISAT synthetic aperture radar images, IEEE T. Geosci. Remote, 51, 2587$2600,2013$. 